\title{
Vorwort zur russischsprachigen Ausgabe
}

Das Hauptziel diese Buches ist eine Beschreibung der Phasengleichgewichte in makround mikroheterogenen Systemen unter strenger Berücksichtigung von Grenzflächenerscheinungen. Im wesentlichen erfolgt dies mit Hilfe der Thermodynamik.

Obwohl die GIBBssche Thermodynamik der Phasengleichgewichte unter Berücksichtigung von Grenzflächenerscheinungen gegenwärtig ein bemerkenswertes Stadium erreicht hat, spiegeln die auf diesem Gebiet vorliegenden Monographien und Utbersichten (darunter auch das relativ vollständige Werk von R. Defay und I. Prigogine „Tension superficielle et adsorption" “, Liege, 1951) ${ }^{1}$ den Inhalt der GibBsschen Originalarbeiten nur unvollständig wider. Bei der Niederschrift dieses Buches war der Autor keineswegs bestrebt, diese Lücke auszufüllen, ist er doch fest davon überzeugt, daß für eine ernsthafte Beschäftigung mit Grenzflächenerscheinungen das Studium des GrBbsschen Werkes notwendig und unumgänglich ist. Daher wurde die Aufmerksamkeit auf die Ausarbeitung jener Probleme gerichtet, die von GrBBs und seinen Schülern entweder nicht oder nur unzureichend behandelt worden sind. Gleichzeitig stellt diese Monographie eine hinreichend vollständige Behandlung der Theorie für Phasengleichgewichte unter Berücksichtigung von Grenzflächenerscheinungen dar, die auch jenen Lesern zugänglich sein dürfte, die nicht im Detail mit den GibBsschen Originalarbeiten vertraut sind.

Für die Darstellung der Grenzflächenerscheinungen wird im vorliegenden Buch als Hauptmethode die Vorstellung von der Grenzflächenschicht endlicher Dicke benutzt. In Verbindung damit, daß gegenwärtig effektive Dicke und Struktur von Grenzflächenschichten zu unmittelbaren Untersuchungsobjekten aufgerückt sind, ist diese Methode der GrBBsschen, in deren Rahmen der Begriff Grenzflächenschichtdicke und somit auch die hiermit verknüpften Eigenschaften von Grenzflächenschichten nicht auftreten, vorzuziehen. Auch vom praktischen Gesichtspunkt ist die Methode der endlichen Schichtdicke vorteilhafter, da sie mit realen, über einen einfachen physikalischen Sinn verfügenden Größen operiert. Dies verringert die Wahrscheinlichkeit von Fehlinterpretationen und erleichtert die Anwendung des ursprünglich für Raumphasen entwickelten thermodynamischen Apparates auf Grenzflächenerscheinungen.

Im Buch treten die strengen thermodynamischen Beziehungen vorwiegend in Form von Differentialgleichungen und -ungleichungen auf. Es sei darauf hingewiesen, da $\beta$ eine Behandlung der Stabilitätsbedingungen und der mit diesen verbundenen thermodynamischen Ungleichungen in der Regel in Monographien zu Grenzflächenerscheinun-

1 Ergänzte Ausgabe: „Surface Tension and Adsorption" von R. Defay, I. Prigogrne unter Mitarbeit von A. Bellemans und D. H. Everetr, ed. by Longmans, Green and Co., 1966. 
gen (auch in dem eingangs erwähnten Werk von Defay und Prigogine) fehlt. Die Analyse dieser Beziehungen führt zu einer Reihe thermodynamischer Gesetzmäßigkeiten, die die Ānderung der Grenzflächeneigenschaften von Lösungen und die Richtung von Adsorptionsprozessen bestimmen. Die Kenntnis dieser Gesetzmäßigkeiten ist für die Untersuchung und praktische Nutzung von Grenzflächenerscheinungen ebenso wertvoll, wie die Kenntnis der Gesetze von Konovalov, Vrevski u. a. für die Betrachtung von Raumphasenprozessen vonnöten ist.

Die abgeleiteten strengen thermodynamischen Beziehungen bilden eine feste Grundlage sowohl für weitere theoretische Untersuchungen als auch für die Anwendung verschiedenartiger Modelle, in denen bestimmte Voraussetzungen über die Struktur von Grenzflächenschichten enthalten sind.

Schließlich sei auf den Gebrauchswert der im vorliegenden Buch betrachteten Beziehungen hingewiesen. Sie enthalten einige auf die Grenzflächenschicht bezogene GröBen, deren experimentelle Bestimmung mit unterschiedlichem Schwierigkeitsgrad behaftet ist, so daß bestimmte Größen ausgehend von anderen berechnet werden können. Daß die Beziehungen in differentieller Form formuliert sind, dürfte für Berechnungen ohne Belang sein, da bei der getroffenen Auswahl der Variablen die Werte aller totalen Differentiale experimentell leicht bestimmbar sind.

Der Autor betrachtet es als angenehme Pflicht, Akademiemitglied P. A. ReHBinder und Prof. K. P. Mršð̌nko seinen Dank für wertvolle Hinweise bei der Durchsicht des Manuskriptes auszusprechen.

A. J. Rusanov 
